\title{
Characterization of pure quantum states of multiple qubits using the Groverian entanglement measure
}

\author{
Yishai Shimoni, Daniel Shapira and Ofer Biham \\ Racah Institute of Physics, The Hebrew University, Jerusalem 91904, Israel
}

\begin{abstract}
The Groverian entanglement measure, $G(\psi)$, is applied to characterize a variety of pure quantum states $|\psi\rangle$ of multiple qubits. The Groverian measure is calculated analytically for certain states of high symmetry, while for arbitrary states it is evaluated using a numerical procedure. In particular, it is calculated for the class of Greenberger-Horne-Zeilinger states, the W states as well as for random pure states of $n$ qubits. The entanglement generated by Grover's algorithm is evaluated by calculating $G(\psi)$ for the intermediate states that are obtained after $t$ Grover iterations, for various initial states and for different sets of the marked states.
\end{abstract}




\section{INTRODUCTION}

In the past decade it was demonstrated that for certain computational tasks, quantum algorithms are more efficient than classical algorithms. Two examples of this effect are provided by Shor's factorization algorithm [1] and Grover's search algorithm [2, 3]. While the understanding of quantum algorithms is still incomplete, there are indications that quantum entanglement plays a crucial role in making them powerful. Quantum algorithms generate entangled states that involve large numbers of qubits. To assess the role of entanglement in the algorithms it would be useful to develop ways to quantify it, which are based on operational considerations. Such considerations in the context of quantum communication between two parties were successfully applied to develop measures of entanglement for bipartite systems [4, 5, 6]. It is thus expected that operational considerations in the context of quantum computation, that involves multiple qubits, may lead to useful entanglement measures for multi-partite systems. Recent work based on axiomatic considerations has provided a set of properties that entanglement measures should satisfy [7, 8, 9, 10]. These properties include the requirement that any entanglement measure should vanish for product states, it should be invariant under local unitary operations and should not increase as a result of any sequence of local operations complemented by only classical communication between the parties. These properties provide useful guidelines in the search for operational measures of entanglement based on quantum algorithms. Measures that satisfy the properties specified above are called entanglement monotones.

Recently, it was shown that the entanglement of a pure quantum state $|\psi\rangle$ of $n$ qubits can be quantified by the utility of this state as an initial state for Grover's search algorithm 11]. Suppose that a given state $|\psi\rangle$ is used as the initial state. However, before starting the search, one is allowed to perform local unitary operations on each qubit in order to maximize the probability, $P_{\max }$, of having a successful quantum search. It was shown that if $|\psi\rangle$ can by transformed to some other state $|\phi\rangle$ by local operations and classical communication then $P_{\max }(\psi) \leq P_{\max }(\phi)$. This observation was used in order to construct the Groverian entanglement measure $G(\psi)$ of pure states. The Groverian measure is an entanglement monotone, and is equivalent to an entanglement measure proposed previously in Refs. [7, 9], which is based on distance measures between $|\psi\rangle$ and the nearest disentangled state.

In this paper we present an explicit representation of $G(\psi)$ and calculate it for a variety 
of pure entangled states using a combination of analytical and numerical methods. The difficulty is that the calculation of the Groverian measure is based on the maximization of a multi-variable function. The number of variables over which the maximization is taken increases with the number of qubits, $n$, in the state $|\psi\rangle$. The measure $G(\psi)$ is calculated analytically for various quantum states that exhibit high symmetry, such as the GreenbergerHorne-Zeilinger state [12] and the $\mathrm{W}$ state [13, 14] of $n$ qubits. The numerical procedure is applied to the calculation of $G(\psi)$ for the states that are produced by Grover's algorithm as a function of the number of iterations, $t$, when the initial state is either a product state or an entangled state and for different sets of marked states. Finally, random states of $n$ qubits are also examined. It is found that homogeneous random sampling of pure states of $n$ qubits produces highly entangled states for which $G(\psi)$ approaches 1 for large $n$.

The paper is organized as follows. The quantum search algorithm, using arbitrary initial states is described in Sec. II. The Groverian entanglement measure is presented in Sec. III. An explicit representation of the Groverian measure is introduced in Sec. IV. This explicit representation is used in Sec. V to perform analytical calculations of the Groverian measure for certain pure states of high symmetry. Numerical calculations of $G(\psi)$ for arbitrary pure states are presented in Sec. VI. The results are summarized in Sec. VII.

\section{QUANTUM SEARCH USING AN ARBITRARY INITIAL STATE}

Consider a search space $D$ containing $N$ elements. We assume, for convenience, that $N=2^{n}$, where $n$ is an integer. In this way, we may represent the elements of $D$ using an $n$-qubit register containing the indices, $i=0, \ldots, N-1$. We assume that a subset of $r$ elements in the search space are marked, that is, they are solutions to the search problem. The distinction between the marked and unmarked elements can be expressed by a suitable function, $f: D \rightarrow\{0,1\}$, such that $f=1$ for the marked elements, and $f=0$ for the rest. The search for a marked element now becomes a search for an element for which $f=1$. To solve this problem on a classical computer one needs to evaluate $f$ for each element, one by one, until a marked state is found. Thus, on average, $N / 2$ evaluations of $f$ are required and $N$ in the worst case. For a quantum computer, on which $f$ to be evaluated coherently, it was shown that a sequence of unitary operations called Grover's algorithm can locate a marked element using only $O(\sqrt{N / r})$ coherent queries of $f$ [2, [3] . 
To describe the operation of the quantum search algorithm we first introduce a register, $|i\rangle=\left|i_{1} \ldots i_{n}\right\rangle$, of $n$ qubits, and an ancilla qubit, $|q\rangle$, to be used in the computation. We also introduce a quantum oracle, a unitary operator $O$ which functions as a black box with the ability to recognize solutions to the search problem. The oracle performs the following unitary operation on computational basis states of the register, $|i\rangle$, and the ancilla, $|q\rangle$ :

$$
O|i\rangle|q\rangle=|i\rangle|q \oplus f(i)\rangle
$$

where $\oplus$ denotes addition modulo 2 . The oracle recognizes marked states in the sense that if $|i\rangle$ is a marked element of the search space, namely $f(i)=1$, the oracle flips the ancilla qubit from $|0\rangle$ to $|1\rangle$ and vice versa, while for unmarked states the ancilla is unchanged. The ancilla qubit is initially set to the state

$$
|-\rangle_{q}=\frac{1}{\sqrt{2}}(|0\rangle-|1\rangle)
$$

With this choice, the action of the oracle is:

$$
O|i\rangle|-\rangle_{q}=(-1)^{f(i)}|i\rangle|-\rangle_{q}
$$

Thus, the only effect of the oracle is to apply a phase of -1 if $|i\rangle$ is a marked basis state, and no phase change if $|i\rangle$ is unmarked. Since the state of the ancilla does not change, one my omit it and write the action of the oracle as $O|i\rangle=(-1)^{f(i)}|i\rangle$.

The original algorithm, as introduced by Grover, includes an initialization stage in which the $n+1$ qubits of the register and the ancilla are prepared in the state $|0\rangle^{\otimes n}|0\rangle_{q}$. Then, the following procedure is performed: a Hadamard gate $H=\frac{1}{\sqrt{2}}\left(\begin{array}{cc}1 & 1 \\ 1 & -1\end{array}\right)$ is applied on each qubit in the register, and the gate $H X$ on the ancilla, where $X=\left(\begin{array}{ll}0 & 1 \\ 1 & 0\end{array}\right)$ is the NOT gate. The matrices are expressed in the computational basis $(|0\rangle,|1\rangle)$. The resulting state is:

$$
|\eta\rangle|-\rangle_{q}
$$

where

$$
|\eta\rangle=\frac{1}{\sqrt{N}} \sum_{i=0}^{N-1}|i\rangle .
$$

The state $\eta$ is considered as the intial state of the algorithm. 
Here we consider a generalized algorithm in which an arbitrary, possibly entangled state

$$
|\psi\rangle=\sum_{i=0}^{N-1} a_{i}|i\rangle,
$$

is used as the initial state instead of $|\eta\rangle$. The ancilla is still prepared as before, namely its state is $|-\rangle_{q}$. The algorithm itself consists of $\tau$ repetitions of the following Grover iteration:

1. Apply the oracle, which has the effect of rotating the marked states by a phase of $\pi$ radians. Since the ancilla is always in the state $|-\rangle_{q}$ the effect of this operation may be described by the following unitary operator

$$
I_{f}^{\pi}=\sum_{i=0}^{N-1}(-1)^{f(i)}|i\rangle\langle i|,
$$

acting only on the register.

2. Carry out the following steps: (i) apply the Hadamard gate to each qubit in the register; (ii) Rotate the $|00 \ldots 0\rangle$ state of the register by a phase of $\pi$ radians. This rotation is similar to step 1, except for the fact that here it is performed on a known state. It takes the form

$$
I_{0}^{\pi}=-2|0\rangle\left\langle 0\left|+\sum_{i=0}^{N-1}\right| i\right\rangle\langle i|,
$$

where the second term on the right hand side is the identity operator, denoted by $I$. (iii) Apply the Hadamard gate again on each qubit in the register.

The resulting operation is

$$
-H^{\otimes n} I_{0}^{\pi} H^{\otimes n}=-I+2 H^{\otimes n}|0\rangle\left\langle 0\left|H^{\otimes n}=-I+2\right| \eta\right\rangle\langle\eta| .
$$

When this operator is applied on the state $|\psi\rangle$ it results in the state

$$
-H^{\otimes n} I_{0}^{\pi} H^{\otimes n}|\psi\rangle=\sum_{i=0}^{N-1}\left(2 \bar{a}-a_{i}\right)|i\rangle
$$


where

$$
\bar{a}=\frac{1}{N} \sum_{i=0}^{N-1} a_{i}
$$

Thus, each amplitude is rotated by $\pi$ around the average of all amplitides of the quantum state.

The combined operation on the register in one Grover iteration is described by

$$
U_{G}=-H^{\otimes n} I_{0}^{\pi} H^{\otimes n} I_{f}^{\pi} .
$$

After the completion of $\tau$ Grover iterations, the register is measured in the computational basis. The optimal number of iterations is [3, 15, 16]

$$
\tau=\left\lfloor\left(\frac{\pi}{2}-\sqrt{\frac{r}{N-r}}\right) / \cos ^{-1}(1-2 r / N)\right\rfloor,
$$

or, approximately for $r \ll N$

$$
\tau=\left\lfloor\frac{\pi}{4} \sqrt{\frac{N}{r}}\right\rfloor,
$$

where $\lfloor x\rfloor$ is the largest integer which is smaller than $x$. Using the original initialization process, at this optimal time, a marked state can be found with almfost certainty, or more precisely with probability

$$
P_{\mathrm{s}}(\eta)=1-O\left(\frac{1}{\sqrt{N}}\right)
$$

With this performance, Grover's algorithm was found to be optimal [15], in the sense that it is as efficient as theoretically possible [17]. A variety of applications were developed, in which the algorithm is used in the solution of other problems [18, 19, 20, 21, 22]. The algorithm was also generalized by allowing an arbitrary (but fixed) unitary transformation to take place of the Hadamard transform and an arbitrary phase rotation instead of the $\pi$ inversion [23, 24, 25, 26].

When a general pure state $|\psi\rangle$ is taken as the initial state for Grover's algorithm the success probability is reduced. In this case, the probability $P(t)$ to find a marked state if a measurement is taken after $t$ iterations depends not only on the initial state $|\psi\rangle$ and the 
number of marked states, but also on the specific identity of the set of marked states. For a given choice of the set of maked states, the states $|\psi(t)\rangle$ obtained after $t$ Grover iterations, starting with an arbitrary initial state $|\psi(0)\rangle$, were calculated using recursion equations [27]. The optimal time to measure as well as the maximal probability of success were found to depend both on the initial state $|\psi\rangle$ and on the specific choice of the set of marked states. Of course, in a real search, the set of marked states is unknown, although it is assumed that the number of marked states is known. To evaluate the success probability and the optimal time to measure one needs to perform an average over all possible choices of the set of marked states. Under these conditions, the optimal time to measure was found to be equal to $\tau$, namely, after the same number of iterations as for the case in which the initial state is $|\eta\rangle$ [28]. However, the success probability $P_{\mathrm{s}}(\psi)$ is reduced. It can be expressed in terms of the amplitudes of the initial state according to

$$
P_{\mathrm{s}}(\psi)=N|\bar{a}|^{2}+O\left(\frac{1}{\sqrt{N}}\right) .
$$

Thus, the success probability of the search depends only on the first moment of the distribution of the amplitudes and not on higher moments. Moreover, it does not depend on the number of marked states, up to a correction of order $r / N$ [28].

\section{THE GROVERIAN ENTANGLEMENT MEASURE}

Consider $n$ parties sharing a pure quantum state $|\psi\rangle$ of $n$ qubits, where each party is in possession of one qubit. The parties use those particular $n$ qubits to perform a quantum search in the space of $N=2^{n}$ elements. Prior to the search, each party may perform local unitary operations on the qubit in their possession. After they complete the local processing of their qubits, all parties send (or teleport) their qubits to the search processing unit. The only processing available in this unit is Grover's search iterations and the subsequent measurement. Thus, the only way the qubits are allowed to interact is through Grover iterations.

The local pre-processing can be expressed by

$$
V=U_{1} \otimes U_{2} \otimes \cdots \otimes U_{n}
$$


where $U_{k}$ is an arbitrary local unitary gate acting on the $k$ th qubit. The initial state inserted into the Grover iterations is then $V|\psi\rangle$. These local operations are chosen such that the success probability $P_{\mathrm{s}}$ of the algorithm will be maximized. The maximal success probability under these conditions will be

$$
P_{\max }(\psi)=\max _{U_{1}, \ldots, U_{n}} P_{\mathrm{s}}\left(U_{1} \otimes U_{2} \otimes \cdots \otimes U_{n}|\psi\rangle\right) .
$$

It turns out that $P_{\max }(\psi)$ can be used to quantify the entanglement present in the state $|\psi\rangle$. To make this assertion more precise, let us write $P_{\max }(\psi)$ in terms of the operator $U_{G}^{\tau}$ representing $\tau$ Grover iterations. For simplicity consider the case in which there is only a single marked state $|m\rangle$. Since the marked state is unknown, the probability $P_{\max }(\psi)$ should be averaged over all possible identities of $m$, namely $m=0,1, \ldots, N-1$. Performing this average we obtain that

$$
P_{\max }(\psi)=\max _{U_{1}, \ldots, U_{n}} \frac{1}{N} \sum_{m=0}^{N-1}\left|\left\langle m\left|U_{G}^{\tau}\left(U_{1} \otimes U_{2} \otimes \cdots \otimes U_{n}\right)\right| \psi\right\rangle\right|^{2},
$$

where the maximization is over all local unitary operations $U_{1}, \ldots, U_{n}$ on the respective qubits. In order to evaluate $P_{\max }(\psi)$ we recall that

$$
U_{G}^{\tau}|\eta\rangle=|m\rangle+O\left(\frac{1}{\sqrt{N}}\right),
$$

where the second term is a small correction. Multiplying this equation by $\left(U_{G}^{\tau}\right)^{\dagger}$ and taking the Hermitian conjugate gives

$$
\langle m| U_{G}^{\tau}=\langle\eta|+O\left(\frac{1}{\sqrt{N}}\right) .
$$

Substituting into Eq. (19) gives, for a general state $|\psi\rangle$,

$$
P_{\max }(\psi)=\max _{U_{1}, \ldots, U_{n}} \frac{1}{N} \sum_{s=0}^{N-1}\left|\left\langle\eta\left|U_{1} \otimes U_{2} \otimes \cdots \otimes U_{n}\right| \psi\right\rangle\right|^{2}+O\left(\frac{1}{\sqrt{N}}\right) .
$$

However, $|\eta\rangle$ is a product state, so that $U_{1}^{\dagger} \otimes U_{2}^{\dagger} \otimes \cdots \otimes U_{n}^{\dagger}|\eta\rangle$ is another product state. Therefore, the maximization in Eq. (22) may, equivalently, be expressed by

$$
P_{\max }(\psi)=\max _{\left|e_{1}, \ldots, e_{n}\right\rangle}\left|\left\langle e_{1}, \ldots, e_{n} \mid \psi\right\rangle\right|^{2}+O\left(\frac{1}{\sqrt{N}}\right)
$$


where the maximization now runs over all product states, $\left|e_{1}, \ldots, e_{n}\right\rangle=\left|e_{1}\right\rangle \otimes \cdots \otimes\left|e_{n}\right\rangle$, of the $n$ qubits.

In Ref. [11] it was shown that the maximum success probability, $P_{\max }(\psi)$, can be used to define an entanglement measure, the Groverian entanglement, for arbitrary pure multiple qubit states. The Groverian entanglement of a state $|\psi\rangle$ is given by:

$$
G(\psi) \equiv \sqrt{1-P_{\max }(\psi)}
$$

Since $P_{\max }(\psi)$ takes values in the range $0 \leq P_{\max }(\psi) \leq 1$, it follows that $0 \leq G(\psi) \leq 1$. It is clear from the definition that all the states $|\psi\rangle$ that can be reached from $|\psi\rangle$ by local unitary operations share the same measure, given by $G(\psi)$. It is also easy to see that for all product states $G(\psi)=0$. In Ref. 11] it was shown that the Groverian entanglement measure is closely related to an entanglement measure introduced previously in Refs. [7, 9]. This relation was used in order to demonstrate that $G(\psi)$ is an entanglement monotone, namely it cannot be increased by local operations and classical communication. Therefore, the Groverian measure is a good entanglement measure for pure quantum states of multiple qubits.

\section{EXPLICIT REPRESENTATION OF THE GROVERIAN MEASURE}

Consider a pure state

$$
|\psi\rangle=\sum_{i=0}^{N-1} a_{i}|i\rangle
$$

of $n$ qubits, where

$$
|i\rangle=\left|i_{1}, i_{2}, \ldots, i_{n}\right\rangle
$$

and $i_{k}$ is the $k$ th most significant bit of the binary integer $i$. The Groverian entanglement measure $G(\psi)$ is given by Eq. (24), where $P_{\max }(\psi)$ is given by Eq. (23). To obtain an explicit formula for $G(\psi)$ we will consider a general product state of $n$ qubits

$$
|e\rangle=\left|e_{1}\right\rangle \otimes \cdots \otimes\left|e_{n}\right\rangle .
$$


The single qubit states can be represented by

$$
\left|e_{k}\right\rangle=\cos \theta_{k}|0\rangle_{k}+e^{i \varphi_{k}} \sin \theta_{k}|1\rangle_{k}
$$

where $k=1,2, \ldots, n$, and global phases are ignored. Note that our angle $0 \leq \theta_{k} \leq \pi / 2$ is a half of the angle $\theta$ used in the Bloch sphere representation, while $\varphi_{k}$ is in the range $0 \leq \varphi_{k} \leq 2 \pi$.

The product state takes the form

$$
\begin{aligned}
\left|e_{1}\right\rangle \otimes \cdots \otimes\left|e_{n}\right\rangle & =\cos \theta_{1} \ldots \cos \theta_{n}|0 \ldots 0\rangle+ \\
& +\cos \theta_{1} \ldots e^{i \varphi_{n}} \sin \theta_{n}|0 \ldots 01\rangle \\
& +\cdots+ \\
& +e^{i \varphi_{1}} \sin \theta_{1} \ldots e^{i \varphi_{n}} \sin \theta_{n}|1 \ldots 1\rangle .
\end{aligned}
$$

Therefore, the product state $|e\rangle$ can by written in the form

$$
|e\rangle=\sum_{i=0}^{N-1} c_{i}|i\rangle
$$

where the coefficient of the basis state $|i\rangle=\left|i_{1}, i_{2}, \ldots, i_{n}\right\rangle$ is

$$
c_{i}=\prod_{k=1}^{n}\left(\cos \theta_{k}\right)^{\bar{i}_{k}}\left(e^{i \varphi_{k}} \sin \theta_{k}\right)^{i_{k}},
$$

where $\bar{i}_{k}=1-i_{k}$. The overlap between the given state $|\psi\rangle$ and a product state $|e\rangle$ is defined by

$$
\langle e \mid \psi\rangle=\sum_{i=0}^{N-1} a_{i} c_{i}^{*},
$$

where $c_{i}^{*}$ is the complex conjugate of $c_{i}$. The calculation of the Groverian measure involves the the maximization of the function

$$
P\left(\theta_{1}, \ldots, \theta_{n}, \varphi_{1}, \ldots, \varphi_{n}, \psi\right)=\left|\left\langle e_{1}, \ldots, e_{n} \mid \psi\right\rangle\right|^{2}
$$

with respect to the variables $\theta_{k}, \varphi_{k}, k=1, \ldots, n$. Note that for $\theta_{k}=\pi / 4$ and $\varphi_{k}=0$, $k=1, \ldots, n$, this function coincides with the success probability of Grover's algorithm 
starting with the initial state $|\psi\rangle$, given by $P_{\mathrm{s}}(\psi)=|\langle\eta \mid \psi\rangle|^{2}$. The maximal probability of success can now be written as

$$
P_{\max }(\psi)=\max _{\theta_{1}, \ldots, \theta_{n}, \varphi_{1}, \ldots, \varphi_{n}} P\left(\theta_{1}, \ldots, \theta_{n}, \varphi_{1}, \ldots, \varphi_{n}, \psi\right),
$$

up to a correction term of order $1 / \sqrt{N}$, and the maximization is taken in the range $0 \leq$ $\theta_{k} \leq \pi / 2$ and $0 \leq \varphi_{k}<2 \pi$.

The case of states $|\psi\rangle$ in which all the amplitudes $a_{i}$ are real is simpler. For such states, the product state $\left|e_{1}, \ldots, e_{n}\right\rangle$ for which the maximum in Eq. (35) is obtained has real amplitueds as well, namely all the angles $\varphi_{k}=0$ or $\pi$. Therefore, in this case the maximization over $\varphi_{k}$ is reduced to a discrete maximization over the binary choice of $\exp \left(i \varphi_{k}\right)= \pm 1$. This term can be removed by doubling the range of $\theta_{k}$ to $-\pi / 2 \leq \theta_{k} \leq \pi / 2$, thus allowing $\sin \theta_{k}$ to be both positive and negative, for the same value of $\cos \theta_{k}$. Thus, for states $|\psi\rangle$ in which all the amplitudes $a_{i}$ are real

$$
P_{\max }(\psi)=\max _{\theta_{1}, \ldots, \theta_{n}} P\left(\theta_{1}, \ldots, \theta_{n}, \psi\right)
$$

where the maximization is over the range $-\pi / 2 \leq \theta_{k} \leq \pi / 2$ and $\varphi_{k}=0, k=1, \ldots, n$.

\section{ANALYTICAL CALCULATIONS OF THE GROVERIAN MEASURE}

Having found an explicit expression for the Groverian entanglement measure, we can now use it in order to characterize certain quantum states that are encountered in various contexts of quantum computation and communication.

\section{A. Two-qubit states}

Consider a state $|\psi\rangle$ of two qubits in which all the amplitudes are real. According to Eq. (36) we can write the overlap of $|\psi\rangle$ with a tensor product of two single qubit states as

$$
P\left(\theta_{1}, \theta_{2}, \psi\right)=\left(a_{00} \cos \theta_{1} \cos \theta_{2}+a_{01} \cos \theta_{1} \sin \theta_{2}+a_{10} \sin \theta_{1} \cos \theta_{2}+a_{11} \sin \theta_{1} \sin \theta_{2}\right)^{2} .
$$


Using standard trigonometric identities we obtain

$$
P\left(\theta_{p}, \theta_{m}, \psi\right)=\left(\frac{a_{00}-a_{11}}{2} \cos \theta_{p}+\frac{a_{00}+a_{11}}{2} \cos \theta_{m}+\frac{a_{10}+a_{01}}{2} \sin \theta_{p}+\frac{a_{10}-a_{01}}{2} \sin \theta_{m}\right)^{2},
$$

where

$$
\begin{gathered}
\theta_{p}=\theta_{1}+\theta_{2} \\
\theta_{m}=\theta_{1}-\theta_{2} .
\end{gathered}
$$

To obtain $P_{\max }(\psi)$ one needs to maximize $P\left(\theta_{1}, \theta_{2}, \psi\right)$ with respect to $\theta_{1}$ and $\theta_{2}$, or equivalently, with respect to $\theta_{p}$ and $\theta_{m}$. This maximization is done by solving

$$
\begin{aligned}
& \frac{\partial P\left(\theta_{p}, \theta_{m}, \psi\right)}{\partial \theta_{p}}=0 \\
& \frac{\partial P\left(\theta_{p}, \theta_{m}, \psi\right)}{\partial \theta_{m}}=0 .
\end{aligned}
$$

The maximun is found for $\theta_{p}$ and $\theta_{m}$ that satisfy

$$
\begin{aligned}
\cos \theta_{p} & =\frac{a_{00}-a_{11}}{\sqrt{\left(a_{10}+a_{01}\right)^{2}+\left(a_{00}-a_{11}\right)^{2}}} \\
\cos \theta_{m} & =\frac{a_{00}+a_{11}}{\sqrt{\left(a_{10}-a_{01}\right)^{2}+\left(a_{00}+a_{11}\right)^{2}}}
\end{aligned}
$$

Inserting these values into Eq. (38) gives rise to

$$
P_{\max }(\psi)=\frac{1}{2}\left(1+\sqrt{1-4|\operatorname{det} D|^{2}}\right)
$$

where the matrix $D$ takes is given by

$$
D=\left(\begin{array}{ll}
a_{00} & a_{01} \\
a_{10} & a_{11}
\end{array}\right)
$$

Eq. (42) is valid also in case that the amplitudes are complex, however in this case the direct maximization is tedius. This result can be shown using the Schmidt decomposition [29] and 
the fact that $P_{\max }(\psi)$ is equal to the square of the maximal Schmidt coefficient [30, 31]. Therefore, the von Neumann entropy, $S$, of the reduced density matrix can be expressed by 11]

$$
S=-P_{\max } \ln _{2} P_{\max }-\left(1-P_{\max }\right) \ln _{2}\left(1-P_{\max }\right) .
$$

Consider a generalized Bell state of the form

$$
|\psi\rangle=a_{00}\left|00>+a_{11}\right| 11>
$$

Inserting these amplitudes into Eq. (42) we obtain that

$$
P_{\max }(\psi)=\max \left(\left|a_{00}\right|^{2},\left|a_{11}\right|^{2}\right) \text {. }
$$

Therefore, the Bell states $\left|\phi_{ \pm}\right\rangle$, for which $\left|a_{00}\right|=\left|a_{11}\right|=1 / \sqrt{2}$, as well as the two other Bell states, are characterized by $P_{\max }=1 / \sqrt{2}$.

In case that $|\psi\rangle$ is a product state, its amplitudes can be expressed by $a_{i j}=b_{i} c_{j}, i, j=0,1$, where $b_{i}$ and $c_{j}$ are the amplitudes of the two single qubit states that form the state $|\psi\rangle$. Plugging this product into Eq. (42) it is easy to see that for product states $P_{\max }=1$.

\section{B. Multiple-qubit states}

\section{Generalized GHZ states:}

The GHZ state of $n$ qubits takes the form

$$
\left|\psi_{\mathrm{GHZ}}\right\rangle=\frac{1}{\sqrt{2}}(|0 \ldots 0\rangle+|1 \ldots 1\rangle) .
$$

This is a generalization of the Bell state $\left|\phi_{+}\right\rangle$to systems of more than two entangled qubits. These states can be further generalized to a continuous class of states of the form

$$
|\psi\rangle=a_{0}|0 \ldots 0\rangle+a_{N-1}|1 \ldots 1\rangle
$$

where $\left|a_{0}\right|^{2}+\left|a_{N-1}\right|^{2}=1$. In this case the overlap function takes the form

$$
P\left(\theta_{1}, \ldots, \theta_{n}, \varphi_{1}, \ldots, \varphi_{n}, \psi\right)=\left(a_{0} \prod_{k=1}^{n} \cos \theta_{k}+a_{N-1} \prod_{k=1}^{n} e^{i \varphi_{k}} \sin \theta_{k}\right)^{2}
$$


To obtain $P_{\max }(\psi)$ we solve the equations $\partial P\left(\theta_{1}, \ldots, \theta_{n}, \psi\right) / \partial \theta_{k}=0$ and $\partial P\left(\theta_{1}, \ldots, \theta_{n}, \psi\right) / \partial \varphi_{k}=0$ for $k=1, \ldots, n$. The solution is $\theta_{k}=0(\pi / 2), k=1, \ldots, n$, when $\left|a_{0}\right|$ is larger (smaller) than $\left|a_{N-1}\right|$. Therefore, $P_{\max }(\psi)=\max \left\{\left|a_{0}\right|^{2},\left|a_{N-1}\right|^{2}\right\}$ and $G(\psi)$ can be obtained from Eq. (24). Its maximal value is obtained when $\left|a_{0}\right|=\left|a_{N-1}\right|=1 / \sqrt{2}$, where $G\left(\psi_{\mathrm{GHZ}}\right)=1 / \sqrt{2}$, namely independent of the number of qubits.

\section{The $W$ states:}

The $\mathrm{W}$ state of $n$ qubits is the symmetrical superpositions of all the basis states which include a single qubit in the 1 state and all the other qubits in the zero state. It takes the form

$$
\left|\psi_{\mathrm{W}}\right\rangle=\frac{1}{\sqrt{n}} \sum_{k=1}^{n}\left|2^{k-1}\right\rangle .
$$

In the case of two qubits it coincides with the Bell state $\left|\psi_{+}\right\rangle=\frac{1}{\sqrt{2}}(|01\rangle+|10\rangle)$, while in the case of three qubits it takes the form $\frac{1}{\sqrt{3}}(|001\rangle+|010\rangle+|100\rangle)$. The overlap function for these states take the form

$$
P\left(\theta_{1}, \ldots, \theta_{n}\right)=\frac{1}{n}\left(\sum_{k=1}^{n} \sin \theta_{k} \prod_{k^{\prime} \neq k} \cos \theta_{k^{\prime}}\right)^{2} .
$$

Taking derivatives with respect to the $\theta_{k}$ 's we obtain that the maximal value is obtained at $\sin \theta_{k}=1 / \sqrt{n}, \cos \theta_{k}=\sqrt{1-1 / n}, k=1, \ldots, n$. The maximal value is

$$
P_{\max }\left(\psi_{\mathrm{W}}\right)=\left(1-\frac{1}{n}\right)^{n-1}
$$

which converges to $1 / e$ as the number of qubits increases. The Groverian measure then

converges to $G\left(\psi_{\mathrm{W}}\right)=\sqrt{1-1 / e}$ at $n \rightarrow \infty$, which is higher than the value for the GHZ states but lower than 1 . We will now examine a class of strongly entangled states, in which the Groverian measure converges to 1 at $n \rightarrow \infty$.

\section{The balanced states}

Consider a state of an even number, $n$, of qubits, that consists of an equal superposition of all the balanced basis states, namely of all those basis states in which the number of 0 's 
is equal to the number of 1's. This state takes the form

$$
|\psi\rangle=\frac{1}{\sqrt{K}}(|0 \ldots 01 \ldots 1\rangle+\text { Permutations }) .
$$

where the binomial coefficient

$$
K=\left(\begin{array}{c}
n \\
n / 2
\end{array}\right)
$$

is equal to the number of different permutations of $n$ bits, where $n / 2$ of them are in the 0 state and the other $n / 2$ are in the 1 state. The overlap function for this state is

$$
P\left(\theta_{1}, \ldots, \theta_{n}, \psi\right)=\sqrt{K}\left(\cos \theta_{1} \cdots \cos \theta_{n / 2} \sin \theta_{n / 2+1} \cdots \sin \theta_{n}+\text { Permutations }\right) .
$$

Taking the derivatives and solving for $\partial P\left(\theta_{1}, \ldots, \theta_{n}, \psi\right) / \partial \theta_{k}=0$, we obtain that the maximum is at $\theta_{k}=\pi / 4, k=1, \ldots, n$, and

$$
P_{\max }(\psi)=K\left(\frac{1}{2}\right)^{n}
$$

In the limit of $n \rightarrow \infty$ one can use the Stirling approximation, that gives rise to

$$
P_{\max }(\psi) \rightarrow \sqrt{\frac{2}{\pi}} \frac{1}{\sqrt{n}} .
$$

Since $P_{\max }(\psi)$ converges to zero, the Groverian measure $G(\psi)$ of the balanced states converges to 1 as $n \rightarrow \infty$.

\section{NUMERICAL CALCULATIONS OF THE GROVERIAN MEASURE}

\section{A. The numerical procedure}

In order to evaluate the Groverian entanglement measure of a given pure state $|\psi\rangle$ of $n$ qubits one has to find the maximal overlap of this state with any product state with the same number of qubits. The overlap is given by the function $P\left(\theta_{1}, \ldots, \theta_{n}, \varphi_{1}, \ldots, \varphi_{n}, \psi\right)$. This is thus a maximization problem in a $2 n$-dimensional space (which is reduced to $n$ dimensions

if all the amplitudes are real). In general, this problem cannot be solved analytically, and therefore the use of numerical calculations is essential. 
The vector space in which the maximization is performed is

$$
\vec{r}=\left(\theta_{1}, \ldots, \theta_{n}, \varphi_{1}, \ldots, \varphi_{n}\right)
$$

for the general case in which the amplitudes are complex [in the special case of real amplitudes it is reduced to $\left.\vec{r}=\left(\theta_{1}, \ldots, \theta_{n}\right)\right]$. The optimization is done using the steepest descend method, namely

$$
\frac{d \vec{r}}{d t}=C \vec{\nabla} P\left(\theta_{1}, \ldots, \theta_{n}, \varphi_{1}, \ldots, \varphi_{n}, \psi\right)
$$

where $C>0$ is a constant and $\vec{\nabla}=\left(\partial / \partial \theta_{1}, \ldots, \partial / \partial \theta_{n}, \partial / \partial \varphi_{1}, \ldots, \partial / \partial \varphi_{n}\right)$.

For a given initial point, $\vec{r}$, the steepest descent method converge to a nearby local maximum. In order to obtain the global maximum, the calculation is repeated with a large number of random initial points. The largest value among all the local maxima that are reached is then picked as the numerical result for $P_{\max }$.

In general, the function $P\left(\theta_{1}, \ldots, \theta_{n}, \varphi_{1}, \ldots, \varphi_{n}, \psi\right)$ consists of a sum of products of trigonometric functions. Each product includes $n$ sine or cosine functions and is thus periodic in all directions. The number of terms in the sum increases exponentially with $n$, making the maximization problem more difficult. We find that the number of local minima also increases with $n$. Thus, as $n$ increases one needs more calls to the steepest descent program with random initial points.

To exemplify the effect of the maximization process we consider the family of states given by

$$
|\psi\rangle=a_{\eta}|\eta\rangle+a_{\mathrm{GHZ}}\left|\psi_{\mathrm{GHZ}}\right\rangle
$$

where $0 \leq a_{\mathrm{GHZ}} \leq 1$ and since the two states are not orthogonal we obtain from the normalization condition that

$$
a_{\eta}=-\sqrt{2 / N} a_{\mathrm{GHZ}}+\sqrt{1-(1-2 / N) a_{\mathrm{GHZ}}^{2}} .
$$

In Fig. 1 we present the success probability $P_{\mathrm{s}}(\psi)$ (dashed line) of Grover's search with the initial state $|\psi\rangle$ as well as $P_{\max }(\psi)$ (solid line) obtained from the numerical procedure, as a function of $a_{\mathrm{GHZ}}^{2}$, for 12 qubits. The two functions follow the same path, decreasing linearly as $a_{\mathrm{GHZ}}^{2}$ increases, up to $a_{\mathrm{GHZ}}^{2} \simeq 0.65$. This means that for the states in this range the 
success probability cannot be increased by local unitary operations. As the two functions depart, $P_{\max }(\psi)$ starts to increase while $P_{\mathrm{s}}(\psi)$ continues to decrease. The size of the gap between the two functions represents the effect of the maximization process.

In Fig. 2 we show the Groverian measure of the generalized GHZ states [Eq. (48)] of 12 qubits as a function of $\left|a_{0}\right|^{2}$. The numerical results (o) coincide with the analytical results (solid line). The graph is symmetric around $\left|a_{0}\right|^{2}=1 / 2$, where the largest value of $G(\psi)$ is obtained. At this point, for real and positive amplitudes the state $\left|\psi_{\mathrm{GHZ}}\right\rangle$ is obtained.

\section{B. Entanglement during the operation of Grover's algorithm:}

There are indications that entanglement plays an important role in making quantum algorithms more efficient than their classical counterparts. Therefore, it is interesting to see how entanglement is generated during the operation of quantum algorithms. Grover's algorithm is particularly suitable for this study because it consists of a large number of iterations of the same set of operations. Furthermore, for any given initial state $|\psi(0)\rangle$, the amplitudes of the state $|\psi(t)\rangle$, obtained after $t$ Grover iterations, can be calculated analytically [27, 28].

In Fig. 3 we present the Groverian entanglement measure of the states $|\psi(t)\rangle$, obtained after $t=0,1, \ldots, 50$ iterations of Grover's algorithm with $n=12$ qubits for the initial state is $|\psi(0)\rangle=|\eta\rangle$ (dashed line). It increases with the time until it reaches its highest value of $1 / \sqrt{2}$ after 25 iterations. Then it follows the same path downwards reaching zero value after 50 iterations, where the marked state is reached. The case in which the initial state is entangled is also considered. To this end we construct the state

$$
|\psi\rangle=a_{\text {even }}\left|\psi_{\text {even }}\right\rangle+a_{\text {odd }}\left|\psi_{\text {odd }}\right\rangle
$$

where $\left|\psi_{\text {even }}\right\rangle$ is a normalized superposition of all the $N / 2$ basis states that include an even number of 1's, while $\left|\psi_{\text {odd }}\right\rangle$ is a normalized superposition of all the other basis states, that include an odd number of 1's. Apart from the initial state $|\eta\rangle$ that is obtained for

$a_{\text {even }}=1 / \sqrt{2}$, we also show the results for $a_{\text {even }}=0.984$ (dotted line), 0.994 (dashed-dotted line) and 1 (solid line). The last state in this list is the one in which the amplitudes of all the basis states that have an odd number of 1's vanish. This state can be obtained from the GHZ state by applying the Hadamard transform on all the qubits. 
In Fig. 4 we show $G(\psi)$ for the states $|\psi(t)\rangle$ obtained after $t$ Grover iterations where $|\psi(0)\rangle=|\eta\rangle$ for 12 qubits and two marked states. In case that the marked states are $|0\rangle$ and $|N-1\rangle$ (solid line) the resulting state after $\tau$ iterations is $\left|\psi_{\mathrm{GHZ}}\right\rangle$. In case that the two marked states are $|0\rangle$ and $|1\rangle$ (dashed line) the resulting state is a superposition of these two states, which is not entangled. Interestingly, during the first $\tau / 2$ iterations these two functions nearly coincide.

In Fig. 5 we present the Groverian measure of the states $|\psi(t)\rangle$ obtained after $t$ Grover iterations, where $|\psi(0)\rangle=|\eta\rangle$ for 12 qubits and 12 marked states. When the set of marked states consists of $|00 \ldots 01\rangle,|00 \ldots 10\rangle, \ldots,|10 \ldots 0\rangle$, namely the basis states of non-zero amplitudes in the $\mathrm{W}$ state (solid line), the register approaches the $\mathrm{W}$ state after 14 iterations. When the marked states are $|i\rangle, i=0, \ldots, 11$ (dashed line) the resulting state is entangled, but exhibits a smaller value of $G(\psi)$.

\section{Random states of $n$ qubits}

How entangled is a randomly chosen pure quantum state of $n$ qubits? To study this question we pick random pure states and evaluate their Groverian entanglement measure using the numerical procedure.

Consider a random pure state

$$
|\psi\rangle=\sum_{i=0}^{N-1} a_{i}|i\rangle
$$

of $n$ qubits, where $a_{i}=\left|a_{i}\right| e^{i \alpha_{i}}$. Such random states can be obtained as follows. In the first step one draws $N$ numbers, $a_{i}, i=0, \ldots, N-1$, independently from a Gaussian distribution centered at 0 , with a standard deviation $\sigma=1$. These numbers are then normalized according to

$$
a_{i} \rightarrow \frac{a_{i}}{\sqrt{\sum_{j=0}^{N-1}\left|a_{j}\right|^{2}}}
$$

The moduli $\left|a_{i}\right|, i=0, \ldots, N-1$ are then obtained as the absolute values of the $a_{i}$ 's. The arguments $\alpha_{i}, i=0, \ldots, N-1$ are drawn from a homogeneous distribution in the range $[0,2 \pi)$. Due to the properties of the Gaussian distribution, the resulting states are 
distributed randomly and isotropically in the Hilbert space that consists of all the pure states of $n$ qubits.

From the normalization condition we obtain that the second moment of the distribution of the moduli of the amplitudes,

$$
\overline{|a|^{2}}=\frac{1}{N} \sum_{i=0}^{N-1}\left|a_{i}\right|^{2},
$$

satisfies $\overline{|a|^{2}}=1 / N$, namely, its square root is of order $1 / \sqrt{N}$. In the case of the randomly

chosen states, due to the random phases, the average amplitude $\bar{a}$ [given by Eq. (11)] will be of the order of $\bar{a}=1 / N$. Therefore, the probability of success $P_{\mathrm{s}}(\psi)$ of Grover's algorithm using a random state as the initial state will vanish like $1 / N$. Our analysis of these states using the numerical procedure shows that for the random states the effect of the optimization procedure is negligible. As a result, $P_{\max }(\psi)$ also vanishes like $1 / N$. Thus, the entanglement measure $G(\psi) \rightarrow 1$ as the number of qubits increases indicating that the vast majority of the states of multiple qubits are very highly entangled.

\section{SUMMARY AND DISCUSSION}

The Groverian entanglement measure was applied to characterize a variety of pure quantum states of multiple qubits. For certain classes of states of high symmetry, the Groverian measure was calculated analytically. In order to evaluate it for arbitrary states with complex amplitudes, a numerical minimization procedure, based on the steepest descent algorithm was developed. It was used in order to evaluate the amount of entanglement generated by Grover's algorithm for different initial states and for different sets of marked states. It was also shown that the typical pure states of $n$ qubits obtained by random sampling are highly entangled, namely for these states $G(\psi)=1$ up to corrections of order $1 / N$.

In recent years several entanglement monotones were proposed as measures of entanglement of multiple qubits [7, 9, 32, 33, 34, 35]. Unlike the case of pure states of two qubits, in which the von Neumann entropy provides a complete characterization of the entanglement, multiple qubit states support a large number of different measures. As a result there is no meaningful way to compare between the different measures. It seems that the issue of what measure is relevant depends on the specific physical or operational context. The 
actual evaluation of entanglement measures turns out to be a difficult computational problem. This is due to the fact that these measures are typically defined as an extremum of some multi-variable function. A singular result in this context is the explicit formula for the entanglement of formation of mixed states of two qubits, obtained in Refs. [36, 37].

The Groverian measure was originally introduced for the case of a single marked state. Recently, it was shown that the same result for $P_{\max }(\psi)$ is obtained in the case of $r$ marked states, up to a correction of order $r / N$ [28], thus removing the restriction of a single marked state. Grover's algorithm can be generalized by replacing the Hadamard transform by an arbitrary unitary operator $U$ on the $n$ qubits [23, 24, 25, 26]. Using the generalized algorithm to evaluate $P_{\max }(\psi)$, one observes that the same result is obtained as long as the operator $U$ is a tensor product of $n$ unitary single qubit operators. In case that the operator $U$ creates entanglement between the qubits in the register it cannot be used to evaluate the Groverian measure.

Interestingly, the Groverian measure coincides for pure states with the measure proposed by Vedral, Plenio, Rippin and Knight [7, 9]. While that measure also applies in the case of mixed states of multiple qubits, we have not been able to extend the operational interpretation of the Groverian measure beyond the case of pure states [11]. It would be interesting to examine the relevance of the Groverian measure to other quantum algorithms such as Shor's algorithm. Further studies of the Groverian measure and related concepts will hopefully contribute to the understanding of the role of entanglement in making quantum algorithms powerful.

\section{Acknowledgments}

This work was supported by the EU Grant No. IST-1999-11234.

[1] P. Shor, Phys. Rev. A 52, 2493 (1995).

[2] L. Grover, in Proceedings of the Twenty-Eighth Annual Symposium on the Theory of Computing (ACM Press, New York, 1996), p. 212.

[3] L. Grover, Phys. Rev. Lett. 79, 325 (1997).

[4] C. H. Bennett, H. J. Bernstein, S. Popescu, and B. Schumacher, Phys. Rev. A 53, 2046 (1996). 
[5] C.H. Bennett, D.P DiVincenzo, J.A.Smolin and W.K Wootters, Phys. Rev. A 54, 3824 (1996).

[6] S. Popescu and D. Rohrlich, Phys. Rev. A 56, R3319 (1997).

[7] V. Vedral, M.B. Plenio, M.A. Rippin and P.L. Knight, Phys. Rev. Lett. 78, 2275 (1997).

[8] G. Vidal, J. Mod. Opt 47, 355 (2000).

[9] V. Vedral and M.B. Plenio, Phys. Rev. A 57, 1619 (1998).

[10] M. Horodecki, P. Horodecki and R. Horodecki, Phys. Rev. Lett. 84, 2014 (2000).

[11] O. Biham, M.A. Nielsen and T. Osborne, Phys. Rev. A 65, 062312 (2002).

[12] D.M. Greenberger, M.A. Horne, A. Shimony and A. Zeilinger, Am. J. Phys. 58, 1131 (1990).

[13] A. Zeilinger, M.A. Horne and A. Greenberger, NASA Conf. Publ. no. 3135, National Aeronotics and Space Administration, Code NTT, Washington DC, 1997.

[14] W. Dur, G. Vidal and J.I. Cirac, Phys. Rev. A 62, 062314 (2000).

[15] C. Zalka, Phys. Rev. A 60, 2746 (1999).

[16] M. Boyer, G. Brassard, P. Hoyer and A. Tapp, Fortsch. der Phys. 46, 493 (1998).

[17] C.H. Bennett, E. Bernstein, G. Brassard and U. Vazirani, SIAM J. Comp. 26, 1510 (1997).

[18] L. Grover, Phys. Rev. Lett. 79, 4709 (1997).

[19] B.M. Terhal and J.A. Smolin, Phys. Rev. A 58, 1822 (1998).

[20] G. Brassard, P. Hoyer and A. Tapp, in Automata Languages and Programming, edited by K.G. Larsen, S. Skyum and G. Winskel (Springer-Verlag, Berlin, 1998), Vol. 1443, p. 820.

[21] L. Grover, Phys. Rev. Lett. 85, 1334 (2000).

[22] N.J. Cerf, LK. Grover and C.P. Williams, Phys. Rev. A 61, 032303 (2000).

[23] R.M. Gingrich, C.P. Williams and N.J. Cerf, Phys. Rev. A 61, 052313 (2000).

[24] E. Biham, O. Biham, D. Biron, M. Grassl, D. Lidar and D. Shapira, Phys. Rev. A 63, 012310 (2001).

[25] L. Grover, Phys. Rev. Lett. 80, 4329 (1998).

[26] G.L. Long, W.L. Zhang, Y.S. Li and L. Nui, Commun. Theor. Phys. 32, 335 (1999).

[27] E. Biham, O. Biham, D. Biron, M. Grassl and D. Lidar, Phys. Rev. A 60, 2742 (1999).

[28] O. Biham, D. Shapira and Y. Shimoni, Phys. Rev. A 68, 0223XX (2003).

[29] M. A. Nielsen and I. L. Chuang, Quantum computation and quantum information (Cambridge University Press, Cambridge, 2000).

[30] H.N. Barnum, e-print quant-ph/9910072.

[31] G. Vidal, D. Jonathan and M.A. Nielsen, Phys. Rev. A 62, 012304 (2000). 
[32] D.A. Meyer and N.R. Wallach, J. Math. Phys. 43, 4273 (2002).

[33] T.-C. Wei and P.M. Goldbart, quant-ph/0307219.

[34] H. Barnum and N. Linden, e-print quant-ph/0103155.

[35] M.S. Leifer, N. Linden and A. Winter, e-print quant-ph/0308008.

[36] S. Hill and W.K. Wootters, Phys. Rev. Lett. 78, 5022 (1997).

[37] W.K. Wootters, Phys. Rev. Lett. 80, 2245 (1998). 
FIG. 1: The success probability $P_{\mathrm{s}}(\psi)$ of Grover's algorithm using $|\psi\rangle$ as the initial state (dashed line), and the maximal success probability $P_{\max }(\psi)$ (solid line), for $|\psi\rangle=a_{\eta}|\eta\rangle+a_{\mathrm{GHz}}\left|\psi_{\mathrm{GHz}}\right\rangle$, as a function of $a_{\mathrm{GHZ}}^{2}$. The two functions coincide up to $a_{\mathrm{GHZ}}^{2} \simeq 0.65$, while above this point a gap appears. The gap broadens as the GHZ state is approached, demonstrating the effect of the maximization by the local pre-processing.

FIG. 2: Analytical results (solid line) and numerical results (o) for the Groverian entanglement measure of generalized GHZ states [Eq. (48)] as a function of $\left|a_{0}\right|^{2}$. The largest value of $G(\psi)=$ $1 / \sqrt{2}$ is obtained for $\left|a_{0}\right|^{2}=1 / 2$, where the state coincides with the GHZ state.

FIG. 3: The Groverian entanglement measure $G(\psi(t))$ for states that are generated by Grover's algorithm as a function of the number of iterations $t$ for $n=12$ and one marked state, where the initial state is $|\psi(0)\rangle$. The curves were obtained for the states $|\psi(0)\rangle$ given by Eq. (62) with $a_{\text {even }}=1 / \sqrt{2}$, namely the $|\eta\rangle$ state (dashed line), 0.984 (dotted line), 0.994 (dashed-dotted line) and 1, namely the state $H^{\otimes n}\left|\psi_{\mathrm{GHZ}}\right\rangle$ (solid line).

FIG. 4: The Groverian measure for the states $|\psi(t)\rangle$ obtained after $t$ Grover iterations where $|\psi(0)\rangle=|\eta\rangle$, for 12 qubits and two marked states. In case that $|0\rangle$ and $|N-1\rangle$ are marked (solid line) the resulting state after $\tau$ iterations is $\left|\psi_{\mathrm{GHZ}}\right\rangle$. If, instead, the marked states are $|0\rangle$ and $|1\rangle$ (dashed line), the register approaches a superposition of these two states, which is not entangled.

FIG. 5: The measure $G(\psi(t))$ for states that are generated by Grover's algorithm as a function of the number of iterations $t$ for $n=12$ and 12 marked states, where the initial state is $|\eta\rangle$. When the marked states are $|00 \ldots 01\rangle,|00 \ldots 10\rangle, \ldots,|10 \ldots 0\rangle$ (solid line), the state obtained after 14 iterations is the $\mathrm{W}$ state (up to a tiny correction due to the discrete nature of the iterations). When the marked states are $|i\rangle, i=0, \ldots, 11$ (dashed line), the resulting state is not as strongly entangled. 


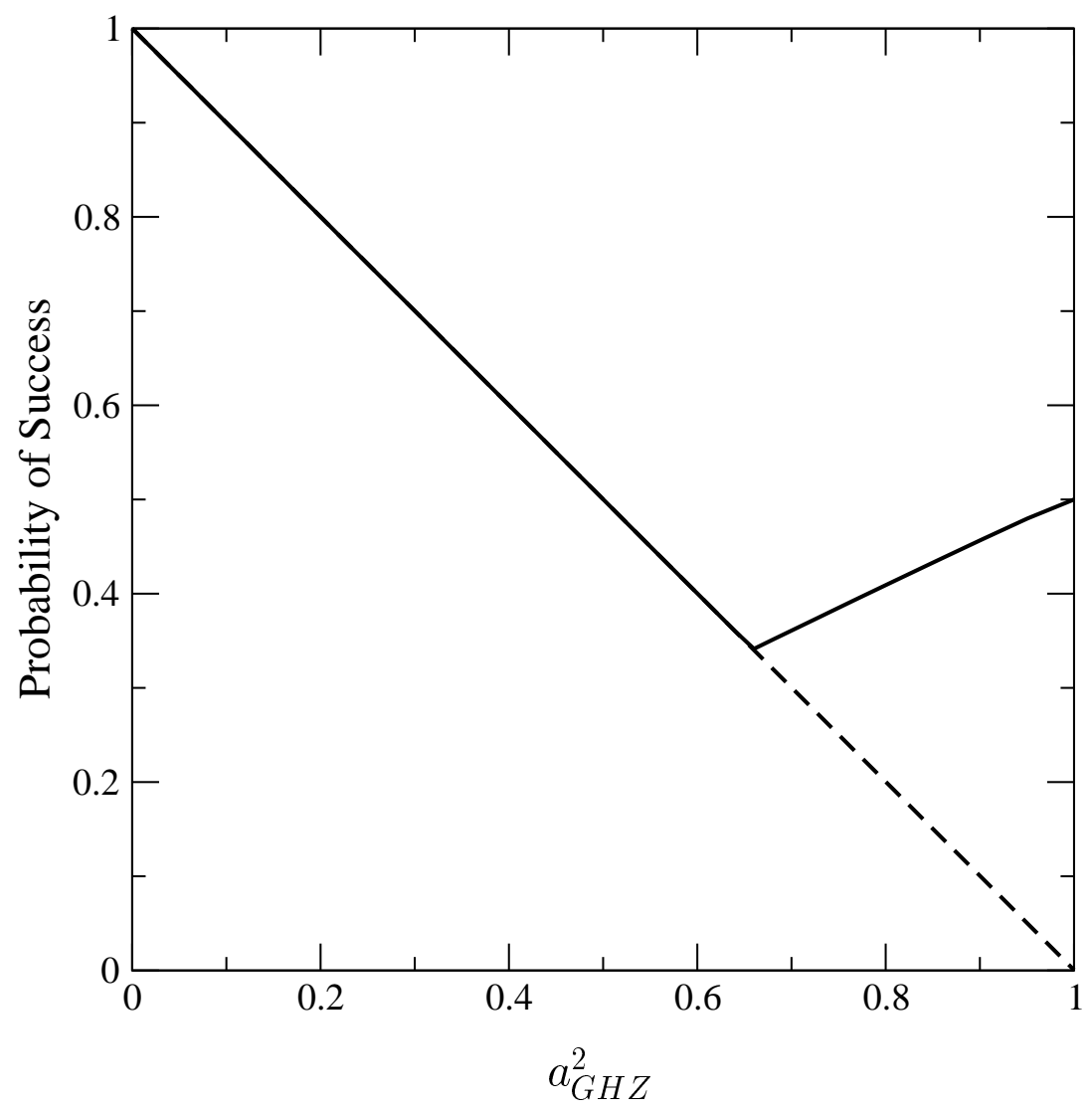

Fig. 1 


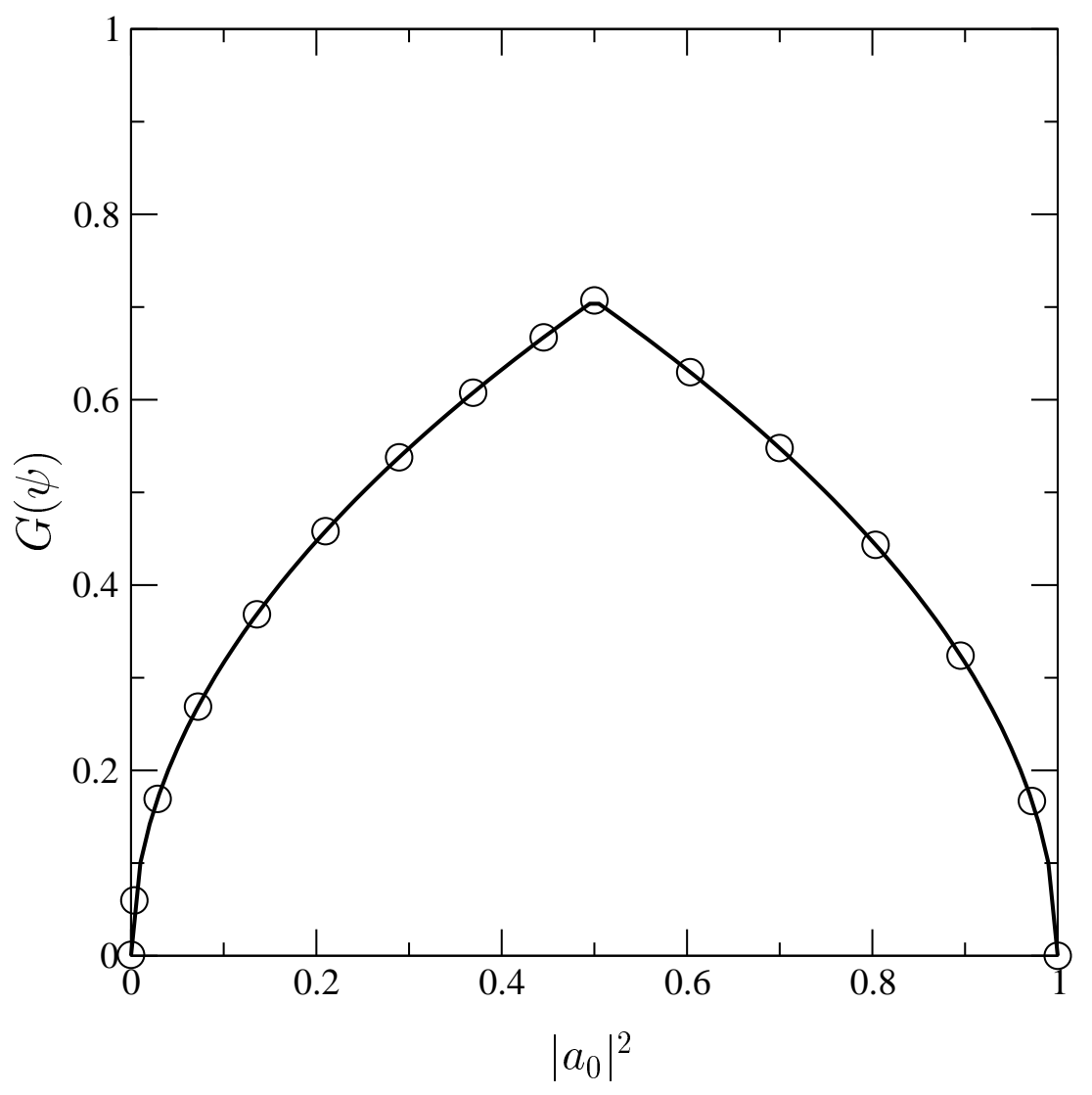

Fig. 2 


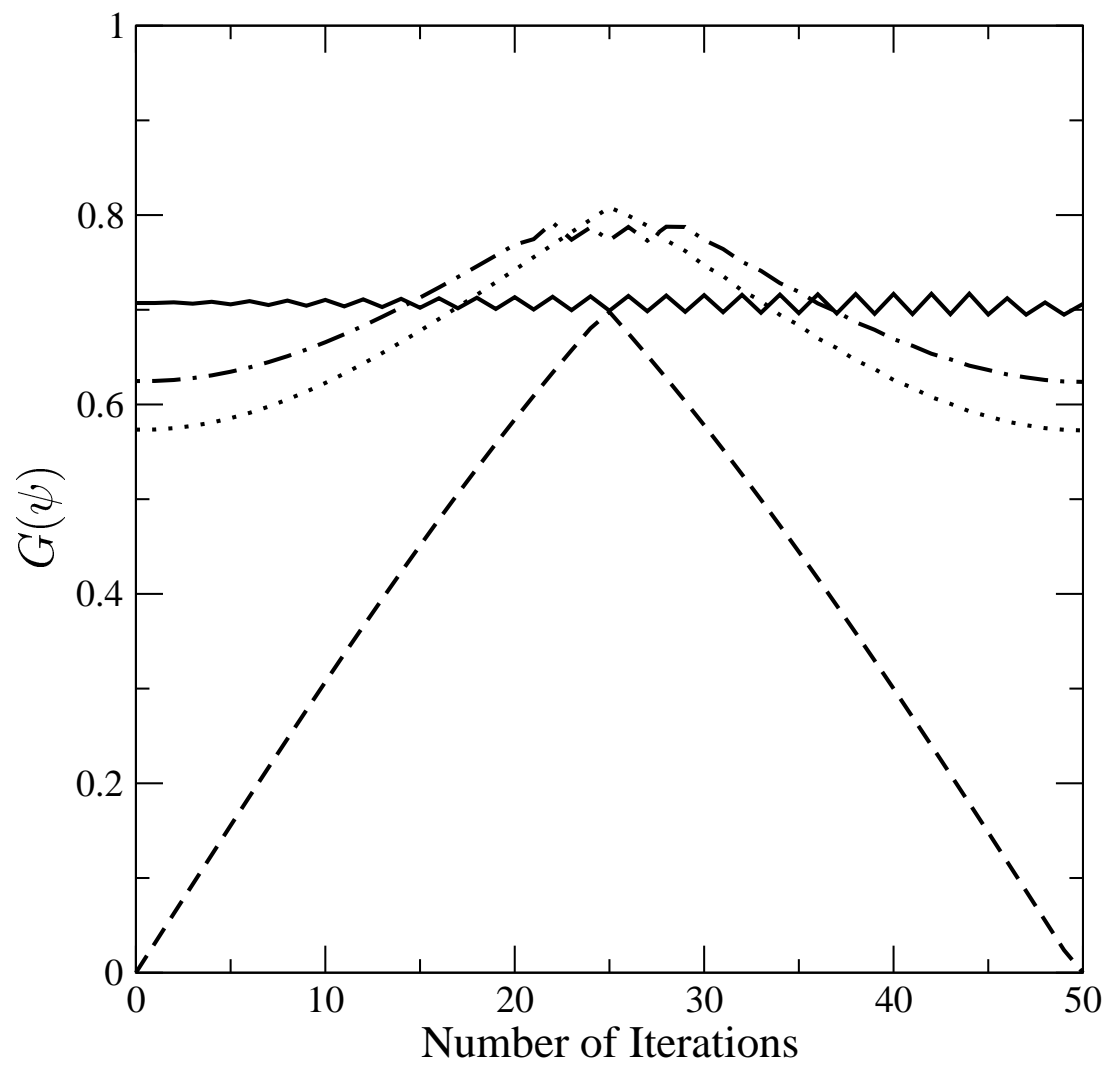

Fig. 3 


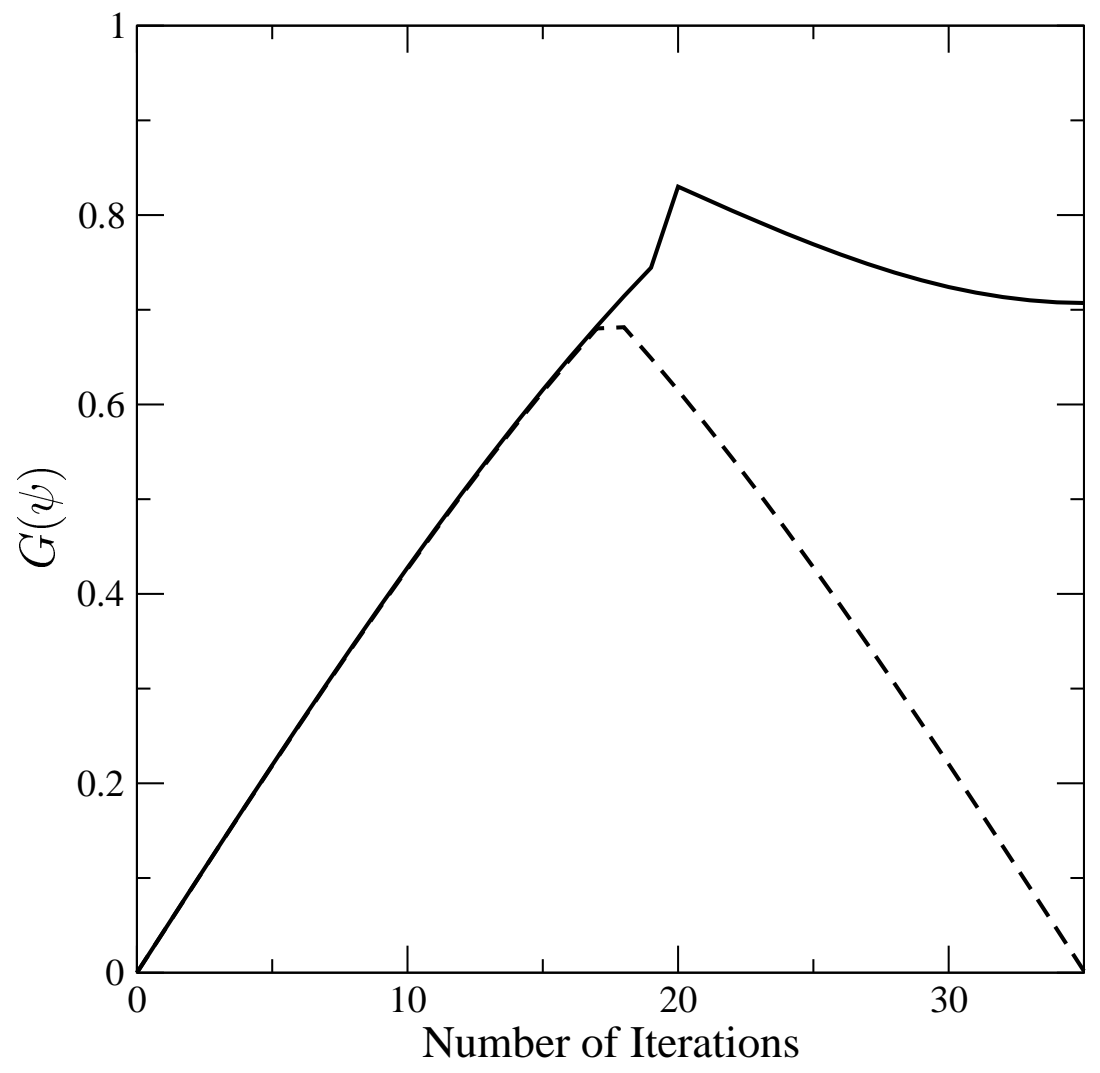

Fig. 4 


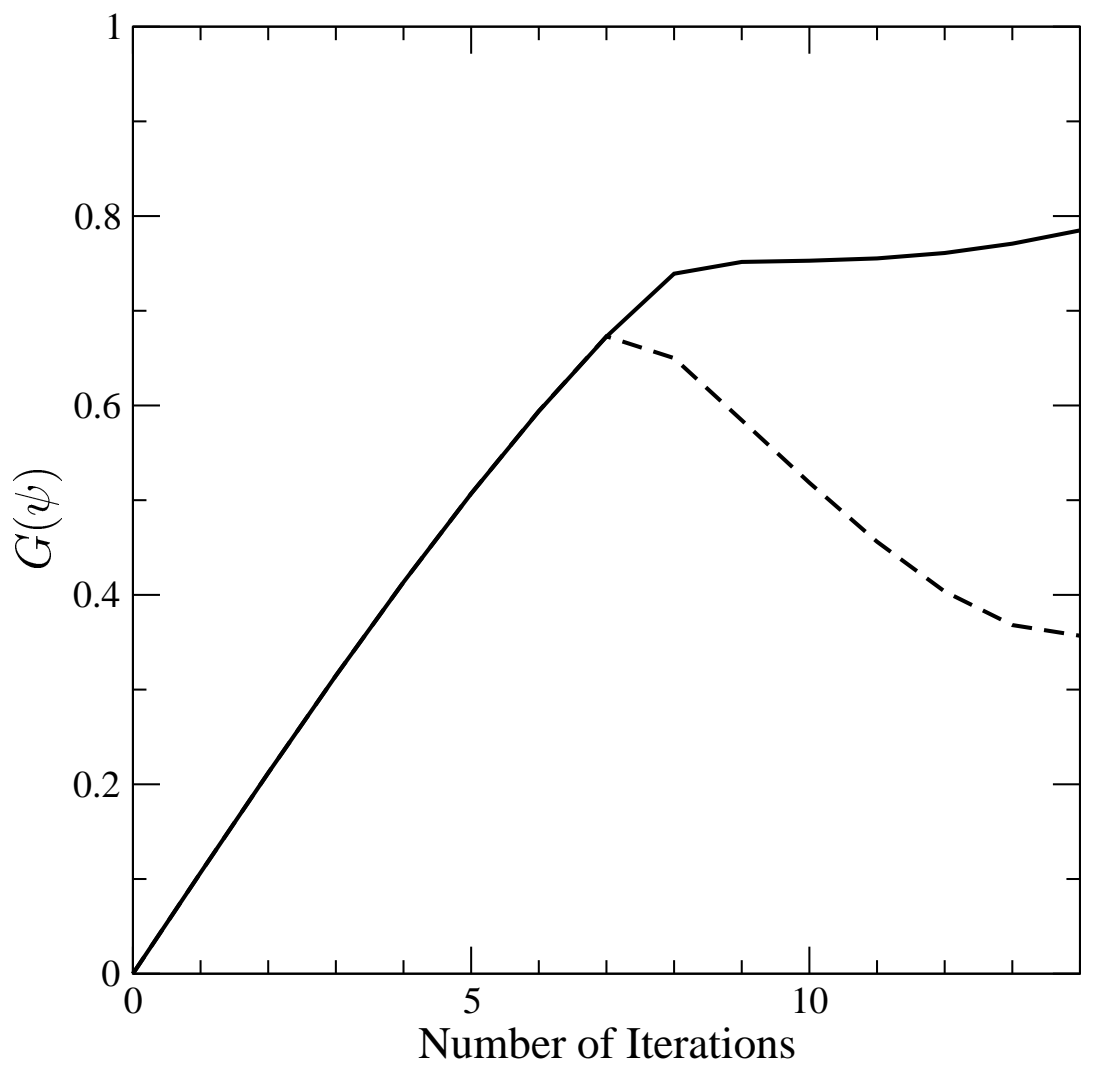

Fig. 5 